\title{
HRJ
}

V.3 n.15 (2022)

Recebido: 21/01/2022

Aceito: 07/03/2022

\section{Diagnósticos de enfermagem em pacientes com Diabetes Mellitus tipo 1}

\author{
Vaneça da Silva Moreira Magalhães ${ }^{1}$ \\ Danyelle Lorrane Carneiro Veloso ${ }^{2}$ \\ Virginia Cunha de Almeida ${ }^{3}$ \\ Ione Batista Nunes Lacerda ${ }^{4}$
}

\author{
${ }^{1}$ Enfermeira Residente do Programa de Residência Multiprofissional em Saúde do Adulto e do Idoso \\ ESCS/FEPECS/SES/DF \\ ${ }^{2}$ Enfermeira Preceptora do Programa de Residência Multiprofissional em Saúde do Adulto e do Idoso \\ ESCS/FEPECS/SES/DF \\ ${ }^{3}$ Enfermeira Tutora do Programa de Residência Multiprofissional em Saúde do Adulto e do Idoso \\ ESCS/FEPECS/SES/DF \\ ${ }^{4}$ Enfermeira Tutora do Programa de Residência Multiprofissional em Saúde do Adulto e do Idoso \\ ESCS/FEPECS/SES/DF
}

\section{RESUMO}

Introdução: O Diabetes Mellitus tipo 1 é uma doença autoimune, decorrente da destruição das células $\beta$ pancreáticas, levando a deficiência completa na produção de insulina. Objetivo: Identificar os principais Diagnósticos de Enfermagem segundo a taxonomia NANDA-I em adultos com DM1. Materiais e Métodos: Estudo de casos múltiplos, descritivo baseado em dados objetivos e subjetivos de pessoas portadoras de DM 1em tratamento insulinoterápico. A coleta de dados ocorreu por meio da aplicação de um questionário e consulta de enfermagem contemplando anamnese e exame físico. Foram identificados os principais Diagnósticos de Enfermagem conforme a taxonomia NANDA-I. Resultados e discussão: Foram avaliados 30 pacientes com diagnóstico médico de DM1. Em relação aos principais Diagnósticos de Enfermagem, foram identificados como mais prevalentes: dor aguda $(66,7 \%)$, integridade tissular prejudicada $(60,0 \%)$, integridade da pele prejudicada $(53,3 \%)$ e conforto prejudicado (50\%). Em relação à quantidade de aplicação de insulina, a maioria relatou realizar mais de 5 aplicações diária.Conclusão: Destaca-se a importância do cuidado de enfermagem sistematizado aos pacientes com DM1, com ênfase nas orientações sobre insulinoterapia e cuidados com a pele, identificando possíveis alterações e prevenindo complicações.

Palavras-chaves: Diagnóstico de Enfermagem; Insulinoterapia; Diabetes Mellitus

Nursing diagnosis in patients with type 1 Diabetes Mellitus

\footnotetext{
ABSTRACT

Introduction: Type 1 Diabetes Mellitus is an autoimmune disease resulting from the destruction of pancreatic $\beta$ cells, leading to a complete deficiency in insulin production. Objective: to identify the main Nursing Diagnoses according to the NANDA-I taxonomy in
} 
adults with DM1. Materials and Methods: Descriptive multiple case study based on objective and subjective data of people with DM 1 undergoing insulin therapy. Data collection occurred through the application of a questionnaire and nursing consultation including anamnesis and physical examination. The main Nursing Diagnoses were identified according to the NANDA-I taxonomy. Results and discussion: 30 patients with a medical diagnosis of DM1 were evaluated. Regarding the main Nursing Diagnoses, the most prevalent were identified as: acute pain (66.7\%), impaired tissue integrity (60.0\%), impaired skin integrity $(53.3 \%)$ and impaired comfort (50\%). Regarding the amount of insulin application, most reported taking more than 5 daily applications. Conclusion: The importance of systematic nursing care for patients with DM1 is highlighted, with emphasis on guidelines on insulin therapy and skin care, identifying possible changes and preventing complications.

Keywords: Nursing Diagnosis; Insulin therapy; Diabetes Mellitus

\section{INTRODUÇÃO}

O Diabetes Mellitus tipo 1 (DM1) é uma doença autoimune, poligênica, decorrente da destruição das células $\beta$ pancreáticas, levando a deficiência completa na produção de insulina $^{1}$. De acordo as estimativas, o Brasil ocupa o terceiro lugar em prevalência em DM1 no mundo ${ }^{2}$. Entre as formas de tratamento do DM1, pode-se mencionar a insulinoterapia, que consiste em um recurso farmacológico para alcançar a estabilidade clínica da doença, na presença de descontrole dos níveis glicêmicos ${ }^{3}$. Ressalta-se, que o uso da insulina é imprescindível no tratamento de DM 1 e deve ser instituído imediatamente após o diagnóstico ${ }^{1}$.

A insulinoterapia consiste na administração de insulina por via subcutânea, quando o paciente possui DM tipo 1 ou não consegue obter controle da glicemia por meio da alimentação, exercícios e agentes antidiabéticos orais ${ }^{4}$. As insulinas industrializadas podem ser classificadas em humanas e análogos de insulina humana. A insulina humana (NPH e Regular) é desenvolvida em laboratório, a partir da tecnologia de DNA recombinante e os análogos são preparações de insulina que sofreram alteração na cadeia de aminoácidos para melhorar o tempo de ação ${ }^{5}$. Entretanto, o uso da insulina pode causar reações adversas aos usuários que a utiliza, principalmente, em longo prazo. A literatura científica aponta para 
possíveis reações adversas, a exemplo de hipoglicemia e reações locais: lipodistrofia, respostas inflamatórias ou infecções, entre outras ${ }^{4}$.

Dessa forma, o paciente, além de lidar com as possíveis complicações do DM, deve seguir um rigor durante a preparação e administração da insulina a fim de evitar complicações em seu quadro clínico. Nesse contexto, vale ressaltar a importância do profissional de enfermagem no que se refere às orientações de saúde, bem como o acompanhamento e auxílio durante o tratamento desses pacientes.

De acordo com Costa et al. ${ }^{6}$ o profissional enfermeiro compartilha informações e trabalha para a conquista do vínculo paciente-profissional, além disso, utiliza abordagens que visam melhorar a comunicação e compreensão, do que se fala, por parte do indivíduo participante, com finalidade de garantir o empoderamento do paciente para o autocuidado e promover uma assistência de enfermagem segura e com qualidade.

A Sistematização da Assistência de Enfermagem (SAE) organiza o trabalho profissional quanto ao método, pessoal e instrumentos, tornando possível a operacionalização do processo de enfermagem ${ }^{7}$.

Nessa perspectiva, para garantir uma assistência sistematizada e de qualidade, os profissionais de enfermagem podem se utilizar do Processo de Enfermagem (PE), um método que compreende diversas etapas, a saber, coleta de dados, diagnóstico de enfermagem, planejamento, estabelecimento de resultados, intervenção e avaliação ${ }^{8}$.

Quanto ao Diagnóstico de Enfermagem (DE), consiste em julgamento clínico sobre uma resposta humana a condições de saúde/processos de vida, ou uma vulnerabilidade a tal resposta, de um indivíduo, uma família, um grupo ou uma comunidade. A NANDA Internacional é uma organização fundamentada, que visa oferecer uma terminologia padronizada de diagnósticos de enfermagem e os apresentam de forma categorizada, a saber, em taxonomia ${ }^{8}$ 
Nesse sentido, o presente estudo tem por objetivo identificar os principais Diagnósticos de Enfermagem segundo a taxonomia NANDA Internacional em adultos com DM1.

\section{MATERIAIS E MÉTODOS}

Trata-se de um estudo de casos múltiplos, descritivo baseado em dados objetivos e subjetivos de pessoas portadoras de DM tipo 1 em tratamento insulinoterápico, atendidas em ambulatório especializado.

Estudo de casos múltiplos consiste em uma estratégia de pesquisa científica, pois permite a investigação de fenômenos no contexto real da enfermagem, com diversas fontes de evidencias, o que torna possível reflexões acerca do fenômeno e sua relação com determinado contexto, a busca de alternativas para a solução de problemas, bem como, contribui para o conhecimento 9 .

O estudo foi realizado na Unidade de Endocrinologia e Diabetes (UENDO) do Hospital Regional de Taguatinga (HRT), o qual faz acompanhamento especializado aos pacientes portadores de DM tipo 1 encaminhados pelas unidades básicas de saúde, conforme protocolo institucional de critérios de encaminhamento, a saber pacientes em uso de insulina em dose otimizada, pacientes com doença renal crônica e/ou pacientes com DM tipo $1^{10}$.

Foram incluídos neste estudo indivíduos com diagnóstico médico de DM tipo 1, idade igual ou superior 18 anos, que fazem uso de insulina há pelo menos 2 anos e que concordaram participar do estudo. Foram excluídos pacientes que fazem uso do Sistema de Infusão Contínua de Insulina (SICI).

A obtenção da amostra ocorreu por conveniência, de acordo o comparecimento ao ambulatório para consulta de enfermagem e orientação quanto à insulinoterapia. 
A coleta de dados ocorreu no período de maio a junho de 2021, por meio da aplicação de um questionário desenvolvido pelas pesquisadoras, abordando questões relativas aos aspectos sociodemográficos, estilo de vida e condições de saúde. Em seguida, realizou-se uma consulta de enfermagem contemplando anamnese e exame físico cefalo-podálico, direcionados por roteiro desenvolvidos pelas pesquisadoras. Destaca-se, que as alterações evidenciadas por meio da anamnese e exame físico, forneceram subsídios para a elaboração dos diagnósticos de enfermagem.

Os dados relativos aos aspectos sociodemográficos, estilo de vida e condições de saúde foram digitados em planilhas eletrônicas do programa Microsoft Excel 2010, em seguida feita a análise descritiva das variáveis com cálculo de frequência absoluta, frequência relativa e medida de tendência central, por meio do programa estatístico Epi Info, versão 7.0 (Centers for Disease Control and Prevention, Atlanta, Estados Unidos).

Realizou-se uma evolução de enfermagem para cada participante, com ênfase nas alterações evidenciadas no exame físico e informações colhidas na anamnese, em seguida foram identificados os principais Diagnósticos de Enfermagem de acordo os domínios da taxonomia NANDA-I, 2018-2020, baseados na Teoria do Autocuidado de Dorothea E. Orem. O Autocuidado é o desempenho e a prática de atividades que os indivíduos realizam em seu benefício para manter a vida, saúde e o bem-estar. Para tanto, há três categorias de requisitos para o autocuidado, a saber: universal, desenvolvimento e desvio de saúde ${ }^{11}$.

Foram identificados os Diagnósticos de Enfermagem, Fatores Relacionados e Características Definidoras. Posteriormente, realizou-se o cálculo de frequência absoluta e relativa para cada $\mathrm{DE}$.

Quanto aos aspectos éticos, o estudo foi apreciado e aprovado pelo Comitê de Ética e Pesquisa da Secretária de Saúde do Distrito Federal (CEP/SES-DF), sob Certificado de Apresentação para Apreciação Ética - CAAE: 44357821.1.0000.5553, com o número de 
parecer 4.688.141. Destaca-se que os indivíduos que aceitaram participar assinaram o Termo de Consentimento Livre e Esclarecido (TCLE).

\section{RESULTADOS E DISCUSSÃO}

Foram avaliados 30 pacientes com diagnóstico médico de DM1. Na população estudada, de maneira geral, houve predomínio do sexo feminino $(66,7 \%)$, na faixa etária de até 35 anos de idade $(53,3 \%)$, não casados $(53,3 \%)$, com escolaridade a partir do ensino médio (90\%). Em relação ao tempo de diagnóstico de DM 1, a maioria foi diagnosticada há menos de 15 anos $(53,3 \%)$, conforme visualizado na tabela 1.

Tabela 1. Características da população do estudo, segundo variáveis sociodemográficas e relacionadas ao DM 1. Taguatinga, Distrito Federal, Brasil, 2021.

\begin{tabular}{lcc}
\hline Variáveis & População total do estudo \\
& $\mathbf{N}$ & \% \\
\hline Sexo & 20 & 66,7 \\
Feminino & 10 & 33,3 \\
Masculino & & \\
Idade & 16 & 53,3 \\
Até 35 anos & 14 & 46,7 \\
Mais de 35 anos & & \\
Estado conjugal & 14 & 46,7 \\
Casados & 16 & 53,3 \\
Não casados & & \\
Escolaridade & 3 & 10,0 \\
Até o ensino fundamental & 27 & 90,0 \\
A partir do ensino médio & & \\
Tempo de diagnóstico & 16 & 53,3 \\
Até 15 anos & 14 & 46,7 \\
Mais de 15 anos &
\end{tabular}

Fonte: Banco de dados do projeto de pesquisa Diagnósticos de Enfermagem em pacientes em insulinoterapia.

Em relação à quantidade de aplicação de insulina diária, a maioria relatou realizar mais de 5 (cinco) aplicações, conforme disposto na tabela 2. 
Tabela 2. Prescrição de insulina descrita de acordo o número de aplicação diária em pacientes com DM1. Taguatinga, Distrito Federal, Brasil, 2021.

\begin{tabular}{c|l|l}
\hline Aplicações diárias de insulina & $\mathbf{N}$ & \% \\
\hline $2-4$ & 13 & 43,3 \\
$5-7$ & 15 & 50,0 \\
8 & 2 & 6,7
\end{tabular}

Fonte: Banco de dados do projeto de pesquisa Diagnósticos de Enfermagem em pacientes em insulinoterapia.

Em relação aos principais Diagnósticos de Enfermagem, foram identificados com maior frequência os seguintes diagnósticos: dor aguda (66,7\%), integridade tissular prejudicada $(60,0 \%)$, integridade da pele prejudicada $(53,3 \%)$ e conforto prejudicado $(50 \%)$, conforme disposto na tabela 3 .

Tabela 3. Distribuição dos Diagnósticos de Enfermagem, identificados em pacientes em insulinoterapia, segundo a Taxonomia NANDA-I. Taguatinga, Distrito Federal, Brasil, 2021.

\begin{tabular}{c|c|c}
\hline Diagnóstico de Enfermagem & N & $\mathbf{\%}$ \\
\hline Dor aguda & 20 & 66,7 \\
Integridade tissular & 19 & 63,3 \\
$\quad$ prejudicada & 16 & 53,3 \\
Integridade da pele & & \\
$\quad$ prejudicada & 15 & 50,0 \\
Conforto prejudicado & 13 & 43,3 \\
$\quad$ Insônia & 12 & 40,0 \\
Disposição para melhora do & 11 & 36,7 \\
autocuidado & 10 & 33,3 \\
Estilo de vida sedentário & & \\
Risco de glicemia instável &
\end{tabular}

Fonte: Banco de dados do projeto de pesquisa Diagnósticos de Enfermagem em pacientes em insulinoterapia.

Na tabela 4, podem ser observados os principais Diagnósticos de Enfermagem (DE), de acordo o domínio da taxonomia NANDA-I, bem como os Fatores Relacionados (FR) e Características Definidoras (CD) com as respectivas frequências relativas. 
Tabela 4. Características definidoras, fatores relacionados e de risco dos principais diagnósticos de enfermagem, identificados em pacientes em insulinoterapia, de acordo os domínios da taxonomia NANDA-I. Taguatinga, Distrito Federal, Brasil, 2021.

\begin{tabular}{|c|c|c|c|}
\hline Domínio & $\begin{array}{l}\text { Diagnóstico de } \\
\text { Enfermagem }\end{array}$ & Fator Relacionado & Característica Definidora \\
\hline 01. Promoção da saúde & $\begin{array}{l}\text { Estilo de vida } \\
\text { sedentário }\end{array}$ & $\begin{array}{c}\text { Interesse/ motivação } \\
\text { insuficiente para atividade } \\
\text { física }(100 \%) .\end{array}$ & $\begin{array}{l}\text { Média de atividade física diária } \\
\text { inferior a recomendada para idade e } \\
\text { sexo }(100 \%) \text {. }\end{array}$ \\
\hline 02. Nutrição & $\begin{array}{l}\text { Risco de glicemia } \\
\text { instável }\end{array}$ & $\begin{array}{l}\text { Controle insuficiente do } \\
\text { diabetes }(100 \%) \text {. }\end{array}$ & \\
\hline \multirow[t]{2}{*}{ 04. Atividade/repouso } & Insônia & $\begin{array}{c}\text { Estressores }(84,6 \%), \\
\text { desconforto físico }(15,4 \%), \\
\text { ansiedade }(23,1 \%) \text {, pesar } \\
(15,4 \%) \text {, doença crônica } \\
(7,7 \%) .\end{array}$ & $\begin{array}{l}\text { Alteração no padrão de sono } \\
\qquad(100 \%) .\end{array}$ \\
\hline & $\begin{array}{l}\text { Disposição para } \\
\text { melhora do } \\
\text { autocuidado }\end{array}$ & $\begin{array}{l}\text { Desejo de melhorar o } \\
\text { autocuidado }(100 \%) \text {. }\end{array}$ & Estilo de vida saudável (100\%). \\
\hline \multirow[t]{2}{*}{ 11. Segurança/proteção } & $\begin{array}{l}\text { Integridade da } \\
\text { pele prejudicada }\end{array}$ & $\begin{array}{c}\text { Aplicação de insulina } \\
(6,25 \%) \text {, hidratação } \\
(56,2 \%) \text {, pressão sobre } \\
\text { saliência óssea }(18,7 \%) \text {, } \\
\text { nível glicêmico elevado } \\
(12,5 \%), \text { trauma }(6,25 \%) \text {. }\end{array}$ & $\begin{array}{c}\text { Pápula }(12,5 \%) \text {, acne }(6,25 \%), \\
\text { cicatriz em pele }(25,0 \%), \\
\text { escoriações }(25,0 \%) \text {, vermelhidão } \\
(12,5 \%) \text {, "rachaduras em calcâneo" } \\
(6,25 \%) \text {, pele ressecada }(37,5 \%), \\
\text { acantose com textura grosseira } \\
(6,25 \%) \text {, calosidade em calcâneo e } \\
\text { região plantar }(18,7 \%) \text {, dermopatia } \\
\text { diabética }(6,25 \%), \\
\text { hiperqueratoseplantar }(18,7 \%), \\
\text { foliculite }(6,25 \%) \text {, dermatite } \\
\text { atópica }(6,25 \%), \text { hiperpigmentação } \\
(6,25 \%) .\end{array}$ \\
\hline & $\begin{array}{l}\text { Integridade } \\
\text { tissular } \\
\text { prejudicada }\end{array}$ & $\begin{array}{c}\text { Aplicação de insulina } \\
(73,7 \%) \text {, volume de } \\
\text { líquidos excessivo }(5,3 \%), \\
\text { controle insuficiente do } \\
\text { Diabetes }(21,0 \%) .\end{array}$ & $\begin{array}{c}\text { Lipodistrofia }(36,8 \%) \text {, hiperemia } \\
(5,3 \%), \text { hematoma }(52,6 \%), \\
\text { enrijecimento dos dedos MMSS } \\
(5,3 \%) \text {, micose em unhas }(5,3 \%), \\
\text { deformidade osséa }(10,5 \%), \\
\text { alteração na sensibilidade dos } \\
\text { pés }(5,3 \%) .\end{array}$ \\
\hline \multirow[t]{2}{*}{ 12. Conforto } & Dor aguda & $\begin{array}{c}\text { Aplicação de insulina } \\
(95,5 \%) \text {, agente biológico } \\
\text { lesivo }(5,0 \%) .\end{array}$ & $\begin{array}{c}\text { Relato de dor }(80,0 \%) \text {, ardência } \\
(15,0 \%) \text {, queimação local }(5,0 \%) \text {. }\end{array}$ \\
\hline & $\begin{array}{c}\text { Conforto } \\
\text { prejudicado }\end{array}$ & $\begin{array}{c}\text { Controle situacional } \\
\text { insuficiente }(93,3 \%), \\
\text { sintomas relacionado à } \\
\text { doença }(6,7 \%) .\end{array}$ & $\begin{array}{l}\text { Alteração no padrão de sono }(80 \%) \text {, } \\
\text { sensação de desconforto }(20,0 \%), \\
\text { medo }(6,7 \%) \text {, ansiedade }(20,0 \%), \\
\text { choro }(6,7 \%) \text {, prurido }(6,7 \%) \text {. }\end{array}$ \\
\hline
\end{tabular}

Fonte: Banco de dados do projeto de pesquisa Diagnósticos de Enfermagem em pacientes em insulinoterapia.

No domínio promoção da saúde foi identificado o DE estilo de vida sedentário $(36,7 \%)$ que consiste em hábito de vida com baixo nível de atividade física ${ }^{8}$. Sabe-se que o exercício físico prescrito e realizado de maneira adequada, proporciona benefícios aos indivíduos com DM, constituindo uma ferramenta indispensável no manejo metabólico ${ }^{1}$. O Ministério da Saúde recomenda para adultos a frequência mínima de 150 minutos de atividade física 
moderada, ou 75 minutos de atividade física vigorosa por semana ${ }^{12}$. Dessa forma, deve-se encorajar a prática de atividade física pelos pacientes, como parte do tratamento do DM para alcançar o controle glicêmico, além dos outros benefícios para a saúde e bem-estar.

No domínio nutrição foi identificado o risco de glicemia instável que, esteve presente em 33,3\% da amostra, definido como suscetibilidade à variação dos níveis séricos de glicose em relação à faixa normal que pode comprometer a saúde ${ }^{8}$. Foi identificado como fator relacionado, o controle insuficiente do diabetes. A insulina quando administrada erroneamente, por via intradérmica ou via intramuscular, pode ter sua absorção retardada ou acelerada, comprometendo, assim o controle glicêmico ${ }^{1}$. Além disso, outros fatores podem interferir na absorção da insulina e controle da glicemia, a exemplo da lipodistrofia. A aplicação de insulina em regiões com lipohipertrofia pode causar absorção irregular de insulina ${ }^{1}$. Destaca-se, que alguns participantes do estudo, equivalente a 36,8\%, apresentavam lipodistrofia. Ademais, o paciente deve seguir um regime terapêutico associando ao tratamento insulinoterápico, a mudança no estilo de vida, como prática de atividade física e alimentação saudável, para atingir o controle glicêmico.

No domínio atividade/repouso foram identificados os seguintes DEs: insônia (43,3\%), e disposição para melhora do autocuidado $(40,0 \%)$.

A insônia é caracterizada por distúrbio na quantidade e qualidade do sono que afeta o desempenho das atividades da vida diária ${ }^{8}$. No presente estudo teve por FR, estressores, desconforto físico, ansiedade, pesar e doença crônica, caracterizado por alteração no padrão de sono. Corrobora com esse achado, estudo brasileiro que descreveu a prevalência de insônia em adultos e idosos atendidos na Atenção Primária a Saúde verificou-se uma maior frequência de insônia entre indivíduos com algumas comorbidades, entre elas o DM ${ }^{13}$.

O DE disposição para melhora do autocuidado ocorre quando o paciente desenvolve atividades para si mesmo com o objetivo de atingir metas relativas à saúde ${ }^{8}$, caracterizado por 
hábitos de vida saudável. No presente estudo boa parte dos participantes, cerca de $40 \%$, relataram hábitos de vida saudáveis. Ressalta-se a importância de estimular o paciente para melhora do seu autocuidado, despertando assim a autonomia do paciente.

No domínio segurança/proteção identificaram-se os DEs integridade tissular prejudicada $(63,3 \%)$ e integridade da pele prejudicada $(53,3 \%)$.

A integridade tissular prejudicada foi o segundo DE mais predominante no estudo. A integridade tissular prejudicada ocorre em casos de danos em membrana mucosa, sistema tegumentar, fáscia muscular, entre outros sistemas ${ }^{8}$.

Em relação à integridade tissular prejudicada, os fatores relacionados foram aplicação de insulina, volume de líquidos excessivos, trauma e complicações da diabetes. Com destaque para a aplicação de insulina, que esteve presente em 14 participantes que apresentaram o diagnóstico. Sendo as principais CDs, hematomas e lipodistrofia do tipo lipo-hipertrofia.

A lipodistrofia é uma alteração no tecido subcutâneo que surge nos locais de aplicação de insulina, sendo as principais manifestações a lipoatrofia e lipo-hipertrofia. A lipohipertrofia, que consiste no acúmulo de gordura nos locais de aplicação de insulina por via subcutânea, formando nódulos endurecidos sob a pele, estando diretamente relacionado ao reuso de agulhas e a não realização do rodízio de aplicações ${ }^{1}$.

É evidente, que a principal causa desse diagnóstico consiste nas repetidas aplicações de insulina. Nesse sentido, é essencial o papel do enfermeiro no que se refere à orientação da técnica correta da aplicação da insulina, com ênfase no rodízio dos locais, bem como a avaliação da pele e manejo adequado das alterações.

Quanto ao diagnóstico integridade da pele prejudicada, ocorre quando há alteração na epiderme e/ou derme ${ }^{8}$. Nesse diagnóstico o principal fator relacionado foi a hidratação, sendo várias $\mathrm{CD}$, dentre elas, alterações de pele característico do paciente diabético. 
As alterações na pele do paciente diabético ocorrem em decorrência de diversas alterações bioquímicas, estruturais e funcionais ${ }^{14}$. Ainda de acordo os referidos autores, identificou alta prevalência de desidratação em diabéticos, sendo relacionada tanto a alterações na função de barreira da pele quanto a variações na umidade do ambiente. Também foi identificado uma menor atividade das glândulas sebáceas e um desequilíbrio na composição dos lipídios do estrato córneo ${ }^{14}$.

Dessa forma, pacientes diabéticos tendem a ter manifestações cutâneas, devido às alterações de pele provenientes do estado de hiperglicemia.

No domínio conforto, os DE presentes foram os seguintes: dor aguda $(66,7 \%)$ e conforto prejudicado $(50,0 \%)$.

A dor aguda foi o DE mais prevalente em todo o estudo, sendo compreendida como a experiência sensorial e emocional desagradável associada a lesão tissular real ou potencial, ou descrita de tal lesão, de início súbito ou lento, leve a intensa, com duração menor que 3 meses $^{8}$. O principal FR foi a aplicação de insulina, evidenciado por relato de dor, ardência e queimação local.

Dos vinte participantes identificados com esse diagnóstico, apenas um não foi relacionado à aplicação de insulina. Doze, equivalente a 63,2\%, relataram realizar entre 5 a 8 aplicações de insulina por dia. Portanto, o número de aplicações pode interferir na experiência sensorial da dor.

Nos pacientes do estudo, essa dor, ardência ou queimação foi referida durante a aplicação de insulina, que pode estar relacionado ao trauma da agulha, aplicação acidental na derme ou músculo, além das repetidas aplicações no mesmo local. Além disso, há associação da dor com o reuso de agulhas de insulina, tal prática não é adequada e deve ser desencorajada, devendo as agulhas ser utilizadas apenas uma vez, e descartadas após o uso ${ }^{15}$. 
Assim, mais uma vez fica evidente a importância do profissional de enfermagem no sentido de orientar os pacientes sobre a técnica correta de aplicação e uso e descarte adequado dos insumos. Além disso, os serviços de saúde devem assegurar ao usuário o acesso a insumos em quantidade suficiente para o seu tratamento.

O conforto prejudicado é definido como percepção de falta de conforto, de alívio e de transcendência nas dimensões física, psicoespiritual, ambiental, cultural e/ou social ${ }^{8}$. Os fatores relacionados identificados foram os seguintes: controle situacional insuficiente, regime de tratamento e sintomas relacionado a doença, caracterizado por insônia, sensação de desconforto, medo, ansiedade, alteração no padrão de sono, prurido e choro.

A etiologia do conforto prejudicado pode estar relacionado ao acometimento por DM 1 e tratamento insulinoterápico. Uma vez que essa doença requer do paciente autocuidado para atingir as metas glicêmicas, a cada refeição necessita ter condutas que influencia diretamente no seu tratamento, seja pela realização da monitorização glicêmica ou pela aplicação de doses diárias e múltiplas de insulina ${ }^{16}$.

De acordo a Associação Nacional de Atenção ao Diabetes (ANAD), a tarefa de gerenciar os níveis de glicose no sangue e mantê-los dentro da normalidade pode ser desafiadora e estressante, além disso, a preocupação excessiva com o progresso da doença podem desencadear episódios de ansiedade $^{17}$. Assim, o paciente pode ter seu conforto comprometido em virtude do DM1.

Destaca-se que nos demais domínios da taxonomia NANDA-I, não foram identificados diagnósticos de enfermagem, apontando para a necessidade de estudos de associação para melhor entender os diagnósticos de enfermagem em pacientes com DM 1. 


\section{CONCLUSÃO}

Este estudo identificou que os principais Diagnósticos de Enfermagem segundo a taxonomia NANDA Internacional em adultos com DM1 foram relacionados a aplicação de insulina por via subcutânea e alterações de pele causadas pelo Diabetes Mellitus. Os resultados reforçam a importância do cuidado de enfermagem sistematizado aos pacientes com DM1, com ênfase nas orientações sobre insulinoterapia e cuidados com a pele identificando possíveis alterações e prevenindo complicações. Destaca-se a importância da utilização de uma teoria de Enfermagem para fornecer embasamento científico para elaboração dos Diagnósticos de Enfermagem.

São limitações do estudo a amostra limitada e a ausência de análise estatística envolvendo associações entre os diagnósticos, seus fatores relacionados e características definidoras. A identificação dos diagnósticos de enfermagem mais frequente na população de DM1 instrumentaliza a equipe de enfermagem atuante na prática assistencial a prestar cuidados direcionados às principais complicações manifestadas por este grupo. Além disso, o estudo contribui com a comunidade científica corroborando com publicações e fortalecendo a prática de enfermagem baseada em evidências.

\section{REFERÊNCIAS}

1. Sociedade Brasileira de Diabetes. Diretrizes da Sociedade Brasileira de Diabetes 20192020.

2. International Diabetes Federation. IDF Diabetes Atlas Ninth edition 2019. 9a Edição. 2019.

3. Gouveia BLA, Sousa MM, Almeida TCF, Sousa VAG, Oliveira SHS. Crenças relacionadas ao uso de insulina em pessoas com Diabetes Mellitus tipo 2. Rev Bras Enferm 2020; 73(3):e20190029.

4. Dicionário de Administração de Medicamentos na Enfermagem. 11ª Ed. EPUB, 2018. 
5. BD. O que é insulina e tipos de insulina. 2020.[citado 2021Nov 20]. Disponível em:https://www.bd.com/pt-br/our-products/diabetes-care/diabetes-learning-center/insulin$\underline{\text { treatment/what-is-insulin-and-insulin-types }}$

6. Costa DA, Cabral KB, Teixeira CC, Mendes JLL, Rosa RR, Cabral FD. Enfermagem e a Educação em Saúde. Ver Cient Esc Estadual Saúde Pública Goiás "Candido Santiago" 2020; 6(3):e6000012.

7. Conselho Federal de Enfermagem. Resolução 358 de 2009. Dispõe sobre a Sistematização da Assistência de Enfermagem e a implementação do Processo de Enfermagem. 2009. [citado 2021Nov 20]. Disponível em: http://www.cofen.gov.br/resoluo-cofen-3582009_4384.html

8. Herdman TH, Kamitsuru S. Diagnósticos de enfermagem da NANDA-I: definições e classificação 2018-2020. 11ª Edição. Porto Alegre: Artmed, 2018.

9. Gautério-Abreu DP, Ávila JA, Santos SSC, Ilha S, Silva BT. Contribuições do estudo de caso para o cuidado de enfermagem: um relato de experiência. Rev enferm UFPE online 2016; 10(3):1149-54.

10. Brasil. Nota Técnica SEI-GDF n. ${ }^{\circ}$ 5/2018 de 23 de agosto de 2018. Dispõe sobre os critérios de encaminhamento de pacientes para a realização de consulta de Endocrinologia no Adulto. Secretaria de Estado de Saúde do Distrito Federal, 2018.

11. George JB. et al. Teorias de enfermagem: os fundamentos à prática profissional. $4^{\mathrm{a}}$ Edição. Porto Alegre: Artmed; 2000.

12. Brasil. Guia de atividade física para a população brasileira. Ministério da Saúde. Brasília/DF, 2021.

13. Pacini E. Prevalência da insônia e fatores associados em adultos e idosos na atenção primária [Trabalho de Conclusão de Curso]. Passo Fundo (RS): Universidade Federal da Fronteira Sul; 2020. 
14. Morales-Cardona CA, Crizón-Díaz DP. Manifestaciones dermatológicas de la diabetes: clasificación y diagnóstico. Iatreia 2020; 33(3):239-250.

15. Sociedade Brasileira de Diabetes. Recomendação sobre o tratamento injetável do Diabetes: insulinas e incretinas. Posicionamento oficial SBD $n^{\circ}$ 01/2017.

16. Fritzen TM. Morbidades psiquiátricas em pacientes com Diabete Melito tipo 1 no Rio Grande do Sul e seus efeitos sobre o controle metabólico [Dissertação]. Porto Alegre (RS): Universidade Federal do Rio Grande do Sul, 2020.

17. Associação Nacional de Atenção ao Diabetes. Diabetes e ansiedade: qual é a relação? 2019.[citado 2021Nov 20]. Disponível em:https://www.anad.org.br/diabetes-e-ansiedadequal-e-a-relacao/. 